\title{
Biocatalysis. Biological systems for the production of chemicals*
}

\author{
M. Held, A. Schmid', J. B. van Beilen, and B. Witholt \\ Institute of Biotechnology, ETH, CH-8093 Zürich, Switzerland
}

Abstract: Biocatalysis harnesses the catalytic potential of enzymes to produce building blocks and end-products for the pharmaceutical and chemical industry. Located at the interface between fermentation processes and petrol-based chemistry, biotransformation processes broaden the toolbox for bioconversion of organic compounds to functionalized products.

\section{BIOCATALYSIS AND BIOTRANSFORMATION CONNECT PETROL-BASED CHEMICALS AND RENEWABLE CARBON SOURCES}

Improvement of synthetic routes according to the guidelines of green chemistry requires increased reaction specificities, reduction of solvent use and emissions, and the use of renewable resources. At first glance, these measures primarily aim at protection of the environment. However, green chemistry also makes economical sense, due to considerable savings on wastewater treatment, energy use, and organic carbon resources. The latter aspects bear directly on the problems associated with our decreasing fossil organic carbon stocks.

A recently published analysis of the life-time of the organic carbon feed stocks indicates that the maximal production rates of crude oil will be reached in the next 10 to 20 years [1]. Alternative stocks such as Canada's oil tars might be available by then [2], but an increase of the oil price in the next few decades is to be anticipated. Alternative energy sources and new sources for building blocks for chemical synthesis have to be developed. At this point it should be emphasized that a significant share of today's oil production is needed for synthetic chemistry. The EU uses 57 MMT (approx. 8\% of the total consumption of the EU) as organic carbon feedstock [3].

A key technology to facilitate and smooth the necessary transition is the use of renewable resources for the production of chemicals using biocatalytic processes. These processes can be subdivided into biotransformation reactions in which a reaction precursor, renewable or petrol-based, is converted to the desired product, and fermentations that use the carbon source for de novo product synthesis, usually from a renewable carbon source (Fig. 1).

Fermentation processes are commonly used for transformation of renewable carbon to typical "biological" compounds such as alcohols, ketones, vitamins, antibiotics, or amino acids, which are metabolites or dead-end products in the production organism. As a matter of fact, the range of fermentation products is much broader than commonly realized. Compounds usually perceived as non-natural such as, for example, indigo, catechol, hexanoic acid, or poly-( $\beta$ )-hydroxyalkanoates can be produced from renewable carbon as well. However, the biochemical and genetic engineering that is required for the development of mature processes from early observations on the lab-scale is demanding. Therefore, the total number of compounds that are produced by fermentation today is still relatively limited.

Biocatalytic processes employ a different strategy. Precursor molecules are fed to the biocatalyst, which transforms them to the desired compound by a limited number of functionalizing steps (usually one). Carbon and energy required for production of the biocatalyst commonly come from a different, easily metabolizable carbon source such as a sugar. Here, the range of products is not limited by the metabolism of the biocatalyst: non-natural (xenobiotic) precursor molecules can be efficiently trans-

*Pure Appl. Chem. Vol. 72, No. 7, 2000. A special topic issue on green chemistry.

$\dagger$ Current address: F. Hoffmann La Roche Ltd., CH-4070 Basel, Switzerland 


\section{A. Fermentation}

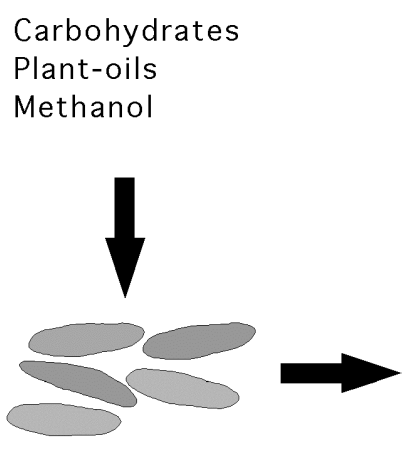

Natural carbon sources are used for production of biomass and for de novo synthesis of products.

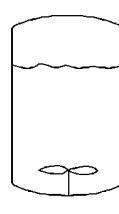

Some classical fermentation products....

$$
\begin{aligned}
& \mathrm{R}-\mathrm{COOH}-\mathrm{OH} \\
& \text { Acids }
\end{aligned}
$$

Vit. $B_{12}$

Vitamins

$$
\mathrm{NH}_{2}-\mathrm{CR}-\mathrm{COOH}
$$

Aminoacids

.. and some not so common products

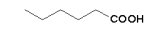

Hexanoicacid
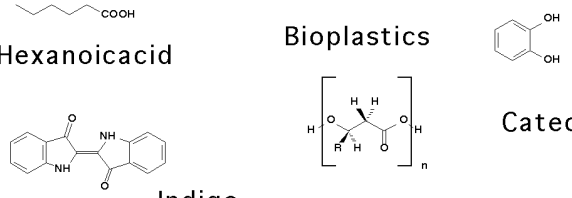

Catechol

\section{B. Biotransformation}

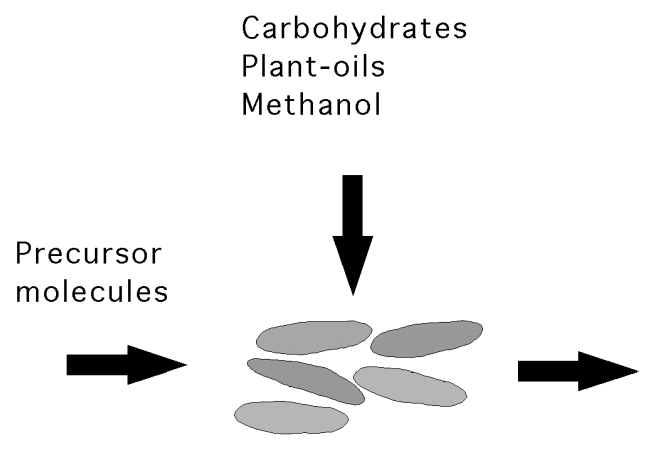

Natural carbon sources are used for the production of the biocatalyst and subsequent transformation of the reaction precursor into the desired product.

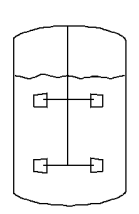

Biotransformation processes can be used for production of numerous fine and specialty chemicals
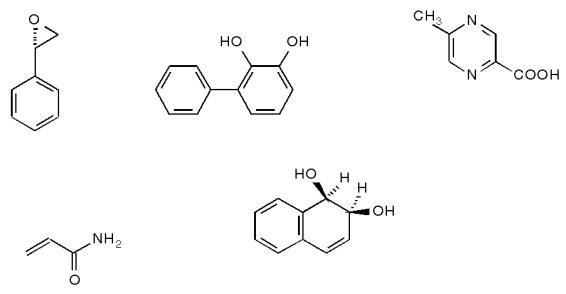

$\bigcup_{\mathrm{COOH}}^{\mathrm{Cl}}$
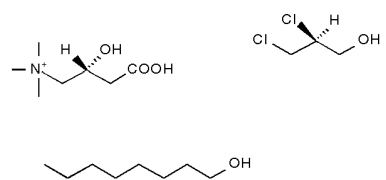

Fig. 1 Biocatalysis. The biotechnological synthesis of end-products and intermediates for the chemical industry.

formed because biocatalysts can transform non-natural as well as natural compounds. The synthesis starts (more than) halfway on the route towards the target molecule.

The research at the Institute of Biotechnology (ETH Zürich) is focused on the development of biotransformation processes that make use of oxygenases. Such biotechnological oxygenation processes are already used on an industrial scale for the production of a variety of high value-added compounds such as $(L)$-carnithine [4a] or cis-cis muconic acid [4b]. The competitiveness of these processes in the present industrial environment is rooted in the high regio and stereo-selectivity of the biocatalysts involved $[5,6]$. 
We expect that tightening of the oil-screw within the next few decades will further strengthen the position of biocatalytic systems; the shuttle function between "biological" and "chemical" compounds and the high reaction specificities favor biocatalytic systems for the modification of raw materials. The detailed knowledge we have gathered on aspects important for biocatalysis-ranging from molecular genetics, enzymology, in vivo and in vitro biotransformations, use and safety of two-liquid phase fermentations, development of integrated processes, and downstream processing-will help to develop new processes.

\section{Enzymes:The key elements for biocatalysis}

Enzymes catalyze an enormous number of reactions that are necessary for the synthesis, modification and degradation, of the organic molecules that make up living organisms, or that are produced by these organisms for their protection and communication. Metabolic pathways and cell growth entail highly diverse reactions such as the formation of carbon-carbon, peptide, or ester bonds, saturation/desaturation of carbon-carbon bonds, and the oxidation by, for instance, oxygen. The corresponding enzymes have been subdivided in six different classes according to their catalytic properties:

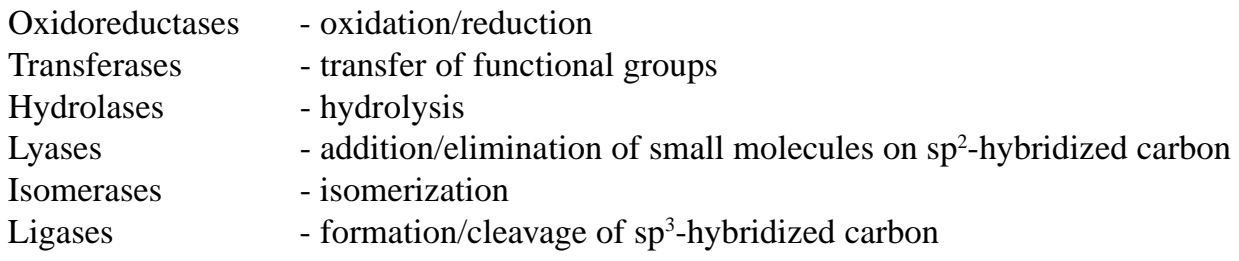

Of the various enzyme classes, hydrolases, which catalyze the cleavage or synthesis of ester and amide-bonds, have found the widest range of applications. Hydrolases are used in laundry detergents (lipases, proteases) and in the food industry (amylases, proteases, lipases, esterases), but also as biocatalysts for the synthesis of chemicals. Applied biocatalysis has the challenging task to make use of the enormous catalytic potential of biological systems for the economical production of fine and bulk chemicals. An example is the multi-tonne scale production of acrylamide from acrylonitrile by a Rhodococcus rhodochrous J1 hydratase (E. C. 4.2.1.84), a reaction that is independent of the metabolic activity of the cells. As a result of the superior kinetics (high specific activity, negligible product, and substrate inhibition) of the hydratase, acrylamide can be formed with space-time yields of up to $100 \mathrm{~g} / \mathrm{L} / \mathrm{h}$ (20 $000 \mathrm{U} / \mathrm{L}$; one unit is defined as a turn-over of one $\mu \mathrm{mol}$ per minute), resulting in product concentrations exceeding $400 \mathrm{~g} / \mathrm{L}$ [7]. The fact that acrylamide can be produced by biotransformation at the $30000 \mathrm{t} / \mathrm{a}$-scale, and the low price difference between acrylonitrile and acrylamide of only $205 \$ / t[8]$ demonstrates the competitiveness of biocatalytic reactions.

Hydrolases have found major uses because it is relatively easy to develop practical applications for these enzymes: they can be formulated as immobilized cell preparations or as partially purified enzyme preparations. In addition, as these enzymes catalyze slightly exergonic equilibrium reactions, they do not require cofactors or complex reaction conditions. Given sufficient enzyme stability, the application of hydrolases in vitro is straightforward. Starting material(s), a (buffered) liquid, and the hydrolytic enzyme-catalyst are the essential requirements to efficiently run a variety of hydrolytic processes. In addition, the direction of the reaction can be reversed by using appropriate reaction conditions (product removal, low water concentration, etc.).

Isomerases are also easy to utilize, but have found few applications, the most important of which is the production of high-fructose corn syrup from glucose using glucose isomerase, a process running at $6000000 \mathrm{t} / \mathrm{a}$. Isomerases are also used to racemize unwanted stereoisomers in amino acid resolutions. In general, lyases are hardly ever applied, as these enzymes generally exhibit narrow substrate ranges.

(C) 2000 IUPAC, Pure and Applied Chemistry 72, 1337-1343 


\section{Specific aspects of the application of oxygenases}

Compared to the simplicity of hydrolysis, isomerization, and to a lesser extent the addition/elimination reactions catalyzed by lyases, the application of oxygenases for biotransformations is highly demanding, and specific features have to be taken into account.

During our work with oxygenases, we identified several factors that have to be considered for the development of competitive biotransformations. In the following section, we will shortly discuss the typical limitations and introduce some recently developed strategies to overcome these bottlenecks:

- Oxygenases mostly require cofactors.

- Very low concentrations of reaction products can poison the biocatalyst.

- Oxygen transfer rates may limit production rates.

Introduction of oxygen by biocatalysts requires (expensive) cofactors

Synthetic reactions carried out by oxidoreductases, transferases and ligases generally require an input in the form of energy-rich functional groups, "redox reagents" (hydrogen, oxygen, electrons) or donors of chemical groups (e.g., methyl). Of these enzymes, the oxidoreductases have the largest potential for biocatalytic applications [7]. Economically interesting reactions are reductions, epoxidations, hydroxylations, and dioxygenations of organic compounds.

Monooxygenases introduce a single oxygen atom derived from molecular oxygen into aliphatic or aromatic organic compounds to form hydroxyl or epoxide groups. The remaining oxygen atom is generally reduced to water with electrons derived from the pyridine cofactors NADH or NADPH. Naturally occurring monooxygenases always require reduction equivalents for the remaining oxygen atom. One exception to this rule is the monooxygenation of naphthalene carried out by a genetically reengineered cytochrome P450 monooxygenase that is also able to use hydrogen peroxide as oxygen-donor. Here, the reaction is independent of reduction equivalents [9]. If this approach can be generalized, it undoubtedly will have a significant impact on industrial-scale biocatalysis.

Dioxygenases introduce both oxygen atoms from molecular oxygen into hydrocarbon substrates to form peroxides. Subsequent reduction to the corresponding alcohol or diol again requires reduction equivalents, derived from $\mathrm{NAD}(\mathrm{P}) \mathrm{H}$. Introduction of an oxo-functionality does not require reduction equivalents, which would be a major advantage in the development of practical processes. Unfortunately, the oxo products formed are typically of low commercial value.

The natural electron donors NADH and NADPH are far too expensive to be used in stoichiometric amounts. Therefore, approaches to recycle $\mathrm{NAD}(\mathrm{P}) \mathrm{H}$ continuously during biocatalytic reactions have been developed. Coupling of the oxygenation reaction to another enzymatically catalyzed reaction that yields $\mathrm{NAD}(\mathrm{P}) \mathrm{H}$ from $\mathrm{NAD}(\mathrm{P})$ is the preferred solution for $\mathrm{NAD}(\mathrm{P}) \mathrm{H}$ recycling. In the case of $\mathrm{NADH}$, an elegant approach to do so is the enzymatic oxidation of formate to carbon dioxide by formate dehydrogenase. In such systems, NADH can be used for more than 80000 cycles, although NADH costs are already negligible after 5000 cycles [10]. Recycling of NADPH can be achieved with glucose dehydrogenase. Here the equilibrium is shifted towards the product because the gluconolactone formed is unstable and hydrolyzes to gluconic acid. Alternative methods to supply reducing equivalents, such as direct chemical reduction, or electrochemical regeneration of cofactor [11], are under development but not mature yet.

For several reasons, industrial-scale processes that make use of oxygenases and oxidoreductases are preferably based on whole-cell biocatalysts. First, the in vitro cofactor regeneration described above is feasible but still relatively expensive. Second, oxygenases often consist of several protein subunits, and the oxygenase component is frequently located in the cell membrane. Purification and reconstitution of such systems in vitro is a challenging task. Both problems can be circumvented by the application of oxygenases in vivo. The (multicomponent) enzyme system is synthesized and assembled by the host cell. No external NAD $(\mathrm{P}) \mathrm{H}$-recycling system is needed, because the host cell regenerates cofactors 
as a part of its normal metabolism. Thus, whole-cell biocatalysts function as miniaturized reaction vessels that take up the substrate, produce a functional enzyme system, convert the substrate to product which diffuses out, and recycle the cofactor.

Clearly, $\mathrm{NAD}(\mathrm{P}) \mathrm{H}$ recycling in vivo requires metabolically active cells; when reducing equivalents are no longer generated, the desired oxygenation reaction also stops. This implies that cells must be maintained in a state that permits a high rate of cofactor regeneration. A minimum requirement for the maintenance of this state is that the cell membrane remains intact during the biocatalytic process; when the membrane permeability increases, low molecular weight metabolites and cofactors are lost. This results in deterioration or even a complete breakdown of intracellular metabolism and cofactor regeneration, even when all relevant enzymes are still fully active. Thus, in developing whole-cell biocatalytic processes, it is important to consider the effects of medium, process conditions, substrates, and products, on cell physiology, enzyme activity, and cofactor regeneration.

\section{Reaction products can poison the biocatalyst}

In their natural habitats, microorganisms usually encounter only low concentrations of growth substrates. Although microorganisms have mechanisms to deal with unfavorable conditions, they cannot handle the high concentrations of non-natural substrates and products that might be used in industrial biocatalytic processes. In fact, most compounds that we study have bactericidal properties and inactivate whole-cell biocatalysts at concentrations in the range of 0.2 to $0.5 \mathrm{~g} / \mathrm{L}$. Therefore, strategies have to be developed to attain high process yields, while maintaining subtoxic substrate and product concentrations. For hydrophobic compounds, this can be accomplished by the addition of an organic, water-immiscible solvent to the reaction mixture [12]. The solvent acts as a bulk-extractant or sink, which efficiently withdraws substrate and product from the water phase, and keeps the effective concentration of these compounds near the biocatalyst at low levels. As the products are concentrated in the organic phase they can be separated easily from the biocatalyst suspension [12]. The extractants can be recycled, and therefore process costs are reduced. As a result, product concentrations of bactericidal compounds such as 1-octanol or $(S)$-styrene epoxide [13] can be increased by at least an order of magnitude to $\sim 20 \mathrm{~g} / \mathrm{L}$. A recently developed two-phase process for production of enantiopure $(S)$-styrene epoxide (99\% ee) from styrene employs recombinant cells of E. coli JM101 (pSPZ10). On the pilot-scale cells formed 3-4.5 g styrene epoxide per L.h at a cell density of 9 to $12 \mathrm{~g}$ CDW per L and a volumetric activity of 400-600 U/L and final process yield of $15 \mathrm{~g} / \mathrm{L}$ [14], respectively.

Continuous product removal can also be accomplished with solid resins. Here, the bioconversion and product extraction is carried out in two separate units. In the bioconversion unit, educt is continuously added at a limiting rate; all added substrate is immediately converted to product, which keeps the substrate concentration low. As the product is formed, it is continuously concentrated in the extraction unit. The solid-phase extraction is performed in an external loop, because the continuous and vigorous stirring of the culture liquid necessary for maximal oxygen transfer damages the solid resins used for product adsorption.

We have recently developed a prototype bioreactor for the production of 3-phenylcatechol with integrated product removal by solid-phase extraction. The overall product concentrations (including product absorbed on the resin) reached $4 \mathrm{~g} / \mathrm{L}$, despite the fact that the substrate, phenylphenol, and the product phenylcatechol are both highly bactericidal at concentrations of only $0.2 \mathrm{~g} / \mathrm{L}$ [15]. Given the broad spectrum of commercially available resins (ion exchange, hydrophobic interaction), this bioprocess technology has considerable potential as both hydrophobic and hydrophilic molecules can be extracted from process liquids under appropriate conditions [16].

\section{Oxygen transfer rates (OTR) limit production rates}

The rate at which oxygen can be transferred to whole-cell biocatalysts in stirred tanks influences biocatalysis rates. The maximum oxygen transfer rate (OTR) in industrial-scale $100-\mathrm{m}^{3}$ continuously stirred tank reactors is in the order of $100 \mathrm{mmole} /(\mathrm{L} . \mathrm{h})$ [17]. How this oxygen is used depends on the 
Table 1 A survey of growth substrates for microorganisms to be used as biocatalysts.

\begin{tabular}{llcc}
\hline Substrate & Glucose & Soybean Oil & Methanol \\
\hline density (t/m3) & & 0.917 & 0.79 \\
heat of combustion (kJ/mole) & 1274 & & 238 \\
MW (g/mole) & 180 & & 32 \\
heat of combustion (GJ/MT) & 7.0 & 40.0 & 7.4 \\
world market price (\$/MT)* & 195 & 396 & 79 \\
& & & $60 * *$ \\
protein yield in SCP processes (\% w/w) [20] & $30-40$ & $\underline{9.9}(35 \%)$ & $\underline{10.7}(38 \%)$ \\
costs per unit energy (\$/GJ) & $\underline{27.9}(100 \%)$ & \\
*prices in Europe at exchange rates of June 1999 & & & \\
**based on gas oil as a comparable carbon source & &
\end{tabular}

biocatalytic system. For in vitro biocatalysis it is theoretically possible to channel all oxygen into the reaction. Under these conditions, the volumetric productivity could amount to $1600 \mathrm{U} / \mathrm{L}$, which is our target for in vitro biocatalysis.

For in vivo whole-cell biocatalysis, oxygen is needed for cell growth and maintenance, as well as for the oxygenation reaction. In practice, more than half of the oxygen fed into the reactor will be used for cellular metabolism. We have previously set a bioconversion rate of $1000 \mathrm{U} / \mathrm{L}$ as a target, based on rates achieved in large-scale industrial aqueous phase fermentations [18]. The styrene production process described above already reached a volumetric activity of 400-600 U/L, indicating that the biocatalyst activity is close to optimal.

Methods to achieve higher production rates may involve measures to increase the OTR, thus increasing theoretical maximal rates. The oxygen partial pressure for example, could be increased by feeding oxygen-enriched air. However, bioprocess safety can be severely compromised, especially if flammable and volatile organic compounds are used as substrates. In addition, oxygen-enriched air is too expensive for routine use as a reagent in biocatalytic reactions, although this might change in the future. If hydrogen is to be used as the primary energy carrier for solar energy, oxygen will be produced as a side-product. Alternatively, oxygen transfer rates can be improved by operating the reactor at higher pressure [18]. However, the increase of the OTR might cause inhibiting concentrations of $\mathrm{CO}_{2}$ due to enhanced cellular respiration [18]. To this end, $\mathrm{CO}_{2}$ formation has to be reduced while maintaining high production rates. For nonfermentative biocatalysts this means that carbon and oxygen are channeled more efficiently in the reaction. The nonproductive fluxes for cell maintenance are reduced, and carbon and oxygen are used more efficiently, which can significantly reduce the overall process costs.

\section{Biocatalytic systems for the transformation of renewable carbon}

The profitability and competitiveness of large-scale/low-margin biocatalytic routes stands and falls with the availability of a cheap carbon source. A rough measure of the economic potential of a substrate for biocatalytic reactions can be made on the basis of the protein yield achieved in single-cell protein (SCP) processes and on the specific energy costs of a particular biodegradable compound (Table 1). Both glucose/molasses and soybean oil are widely used carbon sources: The high cell yield and the low specific energy costs would appear to favor the application of soybean oil. However, soybean oil is difficult to sterilize, can become rancid upon storage, and forms a second organic phase in the reactor, and these aspects are often experienced as disadvantageous for the development of biocatalytic processes.

We believe that the low costs per unit energy, high purity, and the high biomass yield will again make methanol an interesting starting material for biocatalysis in the future. In the early 80s, extensive experience on an industrial scale with methanol as substrate for cultivating microorganism has been

(C) 2000 IUPAC, Pure and Applied Chemistry 72, 1337-1343 
gathered, and recently developed techniques for handling of flammables in biocatalysis [20] suggest that the use of methanol in biocatalytic synthesis should be straightforward. Furthermore, methanol production rates can be expected to increase and its relative price may be further reduced [21] as oil supplies dwindle. Biocatalysis and metabolic engineering will play important roles in the transformation of this simple C1-building block to valuable synthons.

Modern biocatalysis provides new approaches for the generation of building blocks for chemical synthesis and for the production of consumer goods. Increasing oil prices, new cultivation techniques, and R\&D activities on the development of sustainable sources for energy and organic carbon feedstocks can be expected to further improve the competitiveness of biocatalytic routes. The application of naturally occurring or easily synthesized sources of organic carbon and the development of techniques to transform these compounds represent a major challenge for the biotechnological and chemical industries in the coming decades.

\section{REFERENCES}

1. R. A. Kerr. Science 281, 1128-1131 (1998).

2. P. H. Abelson. Science 286, 47 (1999).

3. CEFIC European Chemical Industry Council Facts and Figures 1998, Cap. 5.1 (1998).

4. (a) A. N. Collins, G. N. Sheldrake, J. Crosby. Chirality in Industry II: The commercial manufacture and applications of optically active compounds, Wiley, Chichester, England (1997); (b) A. Liese, K. Seelbach, C. Wandrey. Industrial Biotransformations, Wiley-VCH, Weinheim, Germany (2000).

5. M. G. Wubbolts and B. Witholt. In Pseudomonas, Montie (Ed.), pp. 271-329, Plenum Press, NY, (1998).

6. T. Hudlicky, D. Gonzales, D. T. Gibson. Aldrichimica Acta 32, 35-62 (1999).

7. K. Faber. Biotransformations in Organic Chemistry, 3rd ed., Springer-Verlag, Berlin (1997).

8. J. Hoffman. In Chemical Market Reporter, pp. 22-29, New York (1999).

9. H. Joo, Z. Lin, F. H. Arnold. Nature 399, 670-673 (1999),

10. U. Kragl, W. Kruse, W. Hummel, C. Wandrey. Biotech. Bioeng. 52, 309-319 (1996).

11. W. A. C. Somers, W. v. Hartingsveldt, E. C. A. Stigter, J. P. azelaica v. d. Lugt. Trends Biotechnol. 15, 495-500 (1997).

12. B. Witholt, M. J. de Smet, J. Kingma, J. B. van Beilen, M. Kok, R. G. Lageveen, G. Eggink Trends Biotechnol. 8, 46-52 (1990).

13. M. G. Wubbolts, J. Hoven, B. Melgert, B. Witholt. Enzyme Microb. Technol. 16, 887-894 (1994).

14. S. Panke. PhD thesis, Institute of Biotechnology, Swiss Federal Institute of Technology, Zürich, Switzerland (1999).

15. M. Held, A. Schmid, H.-P. E. Kohler, W. Suske, B. Witholt, M. G. Wubbolts. Biotech. Bioeng. 62, 641-648 (1999).

16. G. L. Lye and J. M. Woodley. Trends Biotechnol. 17, 395-402 (1999).

17. B. Atkinson and F. Mavituna. Biochemical Engineering and Biotechnology Handbook, Stockton Press, New York (1991).

18. A. Schmid. PhD thesis, Institute of Biotechology, Swiss Federal Institute of Technology, Zürich, Switzerland (1997).

19. W. Keim, A. Behr, G. Schmitt. Grundlagen der industriellen Chemie, Otto Salle Verlag, Frankfurt am Main (1986).

20. A. Schmid, A. Kollmer, B. Sonnleitner, B. Witholt. Bioprocess Eng. 20, 91-100. (1999).

21. J. P. Lange. Ind. Eng. Chem. Res. 36, 4282-4290 (1997).

(C) 2000 IUPAC, Pure and Applied Chemistry 72, 1337-1343 